\title{
Instalarse para tomar conciencia histórica del contexto. Aportes pedagógicos sobre la constitución del sujeto, el ciudadano que se configura en el ejercicio de lo cotidiano* Settling oneself taking historical awareness of the context. Pedagogical contributions on the constitution of the self, the cifizen who configures in the exercise of everyday life Ajustando-se para tomar consciência histórica do contexto. As contribuiçoes pedagógicas na constituicãa do sujeito, o cidadão que se configura no exercício do cotidiano
}

\author{
Jorge Hernán Betancourt Cadavid ${ }^{* *}$ \\ (iD http://orcid.org/0000-0002-1387-1708 \\ Universidad de San Buenaventura
}

DOI: http://dx.doi.org/10.21803\%2Fpenamer.10.18.393

Resumen

El recorrido que describe este trabajo se funda en una lectura antropológica filosófica, e histórico-pedagógica sobre la constitución del sujeto, ese que se hace ciudadano en la experiencia diaria de exixtir, dando sentido a todo lo que lo define. Para ello se abordan una diversidad de referentes teóricos que acercan a la obra del profesor Hugo Zemelman***, cuyo trabajo tiene el carácter de faro orientador de la reflexión. El objetivo es contribuir a la construcción de otras miradas que reivindican a los sujetos, a los pueblos y los movimientos olvidados en el contexto nacional, y se proponen contribuciones a una propuesta pedagógica crítica situada, que es el resultado de realidades históricas.

Palabras clave: Colocación, Gnoseología, Sujeto, Subjetividad, Ciudadanía.

Abstract

This paper aims to make an effort on an anthropological-philosophical and historical pedagogical reading about the constitution of the Self, this one who exists on a daily basis experience, giving sense to everything that defined it. For this, a diversity of theoretical approaches lead us close to the work of Hugo Zemelman, which work conduct this thinking. The main objective of this study is to contribute to other views upon the subjects, the people and the forgotten movements from a national context, and it is made contributions to a critical pedagogical proposal that is the result of historical realities.

Key words: Placement, Gnoseology, Subject, Subjectivity, Citizenship.

Resumo

O percurso que descreve este trabalho é baseado em um antropológica leitura filosófica e histórico-pedagógico sobre constituição do sujeito, que um cidadão está em experiência exixtir diária, dando sentido a tudo o que a define. Isso requer uma variedade de quadro teórico para abordar o trabalho do professor Hugo Zemelman, cujo trabalho Tem o caráter de orientar a reflexão da luz. O objetivo é contribuir para a construção de outros pontos de vista que afirmam assuntos, povos e movimentos esquecidos no contexto nacional e contribuições para uma proposta são propostas pedagogia crítica localizado, que é o resultado de realidades históricas.

Palavras-chave: Posicionamento, Epistemologia, Sujeito, Subjetividade, Cidadania.

Cómo referenciar este artículo: Betancourt, J. (2017). Instalarse para tomar conciencia histórica del contexto. Aportes pedagógicos sobre la constitución del sujeto, el ciudadano que se configura en el ejercicio de lo cotidiano. Pensamiento Americano, 10(18), 177188. http://dx.doi.org/10.21803\%2Fpenamer.10.18.393

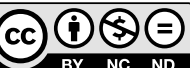

\section{Recibido: Abril 20 de 2016 • Aceptado: Octubre 6 de 2016}

* Artículo de reflexión que presenta resultados de la investigación de Tesis doctoral titulada Una lectura desde la antropología filosófica, e histórico-pedagógica, a la constitución del sujeto y la formación: aportes a la(s) pedagogía(s) crítica(s) en Latinoamérica, trabajo con distinción Cum Laude. Doctorado en Filosofía de la Escuela de Teología, Filosofia y Humanidades de la Universidad Pontificia Bolivariana de Medellín, Colombia.

** Es Doctor en Filosofía. Especialista en Docencia Universitaria. Licenciado en Educación Preescolar. Docente investigador del Grupo Interdisciplinario de Estudios Pedagógicos - GIDEP, en la Facultad de Educación de la Universidad San Buenaventura de Medellín, y del Grupo de Investigación Educación y Subjetividad - GIES de la Facultad de Ciencias Sociales y Educación en la Corporacion Universitaria Lasallista. Docente de Cátedra del Departamento de Pedagogía, en la Facultad de Educación en la Universidad de Antioquia. Jorge.betancourt@usbmed.edu.co jobetancourt@lasallistadocentes.edu.co

*** Hugo Hernán Zemelman Merino nació en octubre de 1931 (Concepción-Chile), murió también en octubre de 2013 en México, país que lo acogió después del golpe militar en 1973. Su actividad académica a través de Latinoamérica se remite de manera especial a la epistemología de los sujetos, entre otros asuntos que sirven de sustento a las Ciencias Sociales en el continente. Fundó y lideró desde 2004 un instituto que tiene por objeto revisar los planteamientos y los modos de pensar para aportar al campo del conocimiento en este continente: IPECAL - "Pensamiento y Cultura en América Latina". Allí se procura el encuentro y la articulación entre personas inquietas con las actuales circunstancias, para que se pueda hacer propio el desafío de la tradición de intelectuales preocupados por instituir autonomía, dignidad, justicia y libertad para los pueblos. 


\section{Introducción: sobre la apropiación del mundo}

El sujeto fundador y activo que buscamos rescatar, puede romper con lo evidente porque anima las formas del lenguaje (Zemelman, 1998, p.49)

Este inicio es en torno a planteamientos del pensar categorial que parte del concepto de realidad y de ubicación en ella. Esa es la posibilidad para el rompimiento de los límites fijados porque genera ámbitos de sentido desde el saber y el pensar situado en un contexto. Aquí hay una proporción entre la conducta y la experiencia, donde la historia aparece como base que reclama ser leída como experiencia de presente. Tales asuntos en conjunción histórica posibilitan un lenguaje pensante para que el que se constituye se confronte a sí mismo en medio de la experiencia de vivir (Adorno, 1992), recuperando el pensar en tanto que se abre a su mundo, y este segundo se abre a él de manera que va acabando con los límites que operan en el razonamiento. Se trata de una ruptura con la lógica y el hecho de tomar distancia de la razón instrumental. Es decir

Cuando hablamos del individuo como de entidad histórica nos referimos no sólo a la existencia espaciotemporal y sensoria de un miembro particular de la especie humana, sino también a la conciencia de su propia individualidad como ser humano consciente, proceso del que forma parte el conocimiento de su identidad. (Horkheimer, 2010, p.143)
Se trata de los detalles que permiten a esta investigación la interpretación pedagógica para el rescate de sujeto como ser pensante y con capacidad de acción, el que se quiere hacer visible conforme la propuesta de Santos (2009). El sujeto y sus espacios son el punto de partida de lo cotidiano que generan movimientos para la construcción del pensamiento. Ahí están precisamente los procesos de subjetivación ${ }^{1}$, dando paso a una discusión epistemológica, donde se da la construcción de ángulos en los que el sujeto usa la historicidad y el pensamiento, lo que está relacionado con la problemática de los campos semánticos y el uso del lenguaje, por lo que se hace importante lograr traducir en un discurso coherente las pretensiones de la realidad, sin que esto signifique restringirse a la constatación de las regulaciones de todo aquello que domina, se trata de un colocarse que permita cuestionar los parámetros desde los cuales se aborda la realidad.

Ahora bien, la historia se encontraba en el estar o existir en un momento, en el mismo hecho de ser un producto en una época determinada. Pero a partir del develamiento de que la historia no es unitaria ${ }^{2}$ y que no hay tal pro-

1 Es importante aclarar que el proceso de subjetivación resulta de las formas a través de las cuales se constituye un sujeto: se trata de los asuntos que construye como conocimiento, sus formas de hacer, vías y caminos que a través del tiempo, estilos y técnicas que elabora en la relación consigo mismo. Estas prácticas que no son para nada sustratos exclusivamente teóricos, son las maneras de hacer en la geografía de lo cotidiano y desde la que el sujeto se ubica, se instala y se apropia históricamente para darse forma.

2 El concepto de historia, la que no es una sola, la que no describe un arrastre homogéneo, es la historia que responde a la noción de discontinuidad. En ella hace presencia la microhistoria de los discursos y las prácticas que transcurren en el anonimato, de escenarios multivariados, cuyos procesos no 
greso indefectible -incluso con la ayuda de la mirada que da la Antropología Histórico Pedagógica-, se plantea la cuestión de cómo es que se produce el individuo históricamente, sin que se pueda encontrar una respuesta en los marcos de una lógica lineal, como la que tuvo lugar desde la Ilustración hasta la actualidad.

La antropología histórico pedagógica y cultural busca poner en perspectivas sus métodos con respecto a su objeto. $\mathrm{Su}$ objetivo ya no es la investigación sobre los hombres o sobre los niños como seres universales, sino más bien sobre los hombres y los niños reales en contextos históricos y sociales determinados en esta perspectiva, la idea de su contexto totalizador del hombre pierde su valor. (Wulf, 2004, p.8)

La realidad que se construye por la necesidad que tiene el sujeto de apropiarla, hace que se comprometa, y este compromiso lo sitúa, lo coloca en momentos históricos determinados. De allí emerge una trama, una episteme en torno a su realidad que prepara el espacio donde se transforma la disposición gnoseológica como postura en voluntad de acción, incorporando al sujeto y su mundo. La realidad es construcción y conformación de una o de diferentes respuestas a opciones de saber y de hacer.

poseen finalidad alguna. Desde esta mirada, el sujeto y los procesos de configuración de su subjetividad, resultan de las relaciones que se producen en lo cotidiano.
El pensar categorial, es decir, el sujeto que se abre al mundo y un mundo que por ende se abre a ese sujeto que se apropia de dicha realidad, reconoce la potencialidad del sujeto de instalarse y apropiarse de lo que lo circunda, construyendo desde su historia, buscando en el sujeto estar determinado (Andrade \& Bedacarratx, 2013).

\section{La Gnoseología ${ }^{3}$ como plataforma en la} configuración del sujeto

La apropiación del mundo asombra al sujeto frente a lo desconocido. Instalarse es un intento por incorporarse a lo indeterminado con un ímpetu que presume de colocarse frente a las costumbres, al yo pensante en forma de sentir, y luego poder pensar. No quiere decir la inexistencia de la objetividad, tanto la acción de los sujetos en la vida cotidiana como la producción científica construyen su propio mundo objetivo o universo simbólico producto de la "matriz de todos los significados objetivados socialmente y subjetivamente reales; toda la sociedad histórica y la biografía de un individuo se ven como hechos que ocurren dentro de ese universo" (Retamozo 7). Se trata de la manera para romper con los límites y elevarse en contra de lo instaurado. Cuestiones que remiten con carácter de prioridad al conoci-

3 La Gnoseología no es exactamente una Epistemología -la segunda de ellas analiza las disciplinas del conocimiento científico-. La Gnoseología se dedica a lo que NO ha sido elevado al estatuto de lo científico, y por ello trabaja sobre el conocimiento general, sobre todo aquello que no se encuentra en el terreno de las ciencias en el sentido positivista. Así que, la Gnoseología asume el conocimiento en general para procurar la delimitación de lo que se define como conocimiento, que no necesariamente tiene que ser científico. 
miento gnoseológico, ya que se trata de ubicar la forma de establecer relaciones de ser y estar del hombre con la realidad de su mundo. Estar, hace referencia al esfuerzo de instalarse en el mundo:

Nuestra generación es testigo y protagonista de múltiples y acelerados cambios. El presente siglo ha sido escenario de profundas transformaciones en todos los órdenes a lo largo y ancho del planeta; cambios que evidencian, el inmenso potencial de la dinámica originada con la expansión capitalista y el proyecto moderno, pero a la vez, sus límites y agotamiento, además, ponen de manifiesto la irrupción de factores y fuerzas nuevas que aún no alcanzamos a comprender plenamente. (Torres, 1994, p.5)

Esta es una dialéctica en la que subyace la construcción del conocimiento, y del sujeto constructor de realidades: cierre-apertura, en la que la lógica del cierre opera en el campo semántico propio del lenguaje de comunicación. La apertura por su parte no tiene la misma lógica, se refiere a los horizontes de sentido, a la posibilidad de relación con base en el desafío de lo indeterminado. Entonces la propuesta sobre el sujeto que emerge de la interpretación hecha en la investigación, es que él se apropia de la realidad desde su capacidad de cimentación... ahí está el instalarse en el mundo, de la apropiación que hace del mismo, el hacerse ciudadano. Instalarse depende de desarrollar la necesidad de realidad como experiencia y con- ciencia que se abre a lo contextual y situado, a su mundo inmediato, que no es solamente la conjugación entre funciones cognitivas, sino desafíos gnoseológicos de una conciencia ética. Instalarse en el mundo es investigar el contexto para la ampliación del sujeto.

Algunos problemas que se anticipan, son el de la articulación de las funciones cognitivas con las de la naturaleza gnoseológica con mayor amplitud, que refleja la multi-dimensionalidad del hombre, y así mismo los desafíos multi-disciplinarios que enfrentan la realidad; además el problema de esta a su vez son las dos dimensiones que pueden ser tomadas, como experiencia vivencial y como conciencia abierta que trasciende a los límites conceptuales establecidos. De la misma manera se hace necesario considerar las necesidades de un lenguaje de significantes que permita instalarse en el mundo en la medida que contribuye a formarse como sujeto en su momento histórico-cultural.

\section{Romper con lo establecido}

Este quiebre es un desafío ético, pero implica retos en la forma de razonar: abrirse gnoseológicamente para recuperarse como sujeto, “... se refiere a un comportamiento activo y define ámbitos de actividad social" (Koselleck, 2012). Se trata de abrir los parámetros impuestos al pensamiento como la función propia del curso categorial (Betancourt \& Rivera, 2009). El mundo globalizado sigue generando una relación de lo humano con su contexto como una realidad externa que lo aleja de sí mismo como 
sujeto (Betancourt, 2012; 2013). El problema inicial es incursionar por horizontes más extensos, y poder recuperarse como sujeto en su propia singularidad, influida y desafiada por esa extensión. Es imperativo recobrar la capacidad de ser consciente y activo.

Es importante insistir en el hecho de que se trata de una actitud racional que libera a la razón como acción del proceso de subjetivación, sin lugar a dudas fundante en el proceso de constitución del sujeto -el concepto de formación desarrollado en Koselleck (2012)-. $\mathrm{El}$ asunto es que no es como fundamento que toma las ideas establecidas por los discursos, sino como forma de la razón misma. Esto corresponde al discurso categorial que consiste en pensar desde significantes que se traducen en un tipo particular de enunciados, como ya se había mencionado antes. Es posible siempre que parta del supuesto de que los límites conceptuales se caracterizan por su movimiento, desafío que va más allá de construir un modo de explicar o de teorizar.

Hemos hablado de la recuperación de la subjetividad en el ámbito de la constituyente, y, desde este ángulo, del sujeto como protagonista. La forma de pensar constitutiva (capaz de romper con los parámetros y abrirse a lo inédito) está, por consiguiente, estrechamente vinculada con la recuperación del sujeto, porque el desafío de potenciar a este se vincula con el rescate de las funciones gnoseológicas latentes en la tradición cultural e intelectual; por ahora reducidas ambas a la función puramente cognitiva. (Zemelman, 1998, p.49)

Los enunciados se fundan en la necesidad del sujeto de entrar en contacto con lo que le rodea. Pero lo importante en el rompimiento con lo establecido, el enfrentamiento con las hegemonías que lo invisibilizan es un esfuerzo del sujeto para situarse ante lo inédito y en medio de todo esto posibilitar los medios de subjetivación que hacen posible la configuración de sí mismo. Este es el esfuerzo de apropiación que se funda en la construcción del ángulo del discurso categorial, lo que ya se ha mencionado como una forma de pensar de los significantes.

\section{Los ángulos de sentido, y la necesidad de} instalarse, de apropiarse

La idea de pensar desde el lenguaje de los significantes, apunta a convertir al lenguaje en el límite del mundo (Benjamin, 2008). El límite descansa en la amplitud de opciones que pueden asumir los sentidos que tienen que usar el pensamiento y su expresión, no solamente para comunicarnos, también para los límites aceptados. Todo lo que se descarta de la conciencia, sale también de la potencia, por tanto, se trata de desarrollar una actitud que permita entender que la autoactividad del sujeto, como resultado de su encuentro con el mundo, consiste en una expansión de su subjetividad.

Todo esto corresponde a la des-parametrización del pensamiento, en cuanto se abren nuevas exigencias de lectura de la realidad po- 
niendo a un lado los parámetros establecidos, así como de repensar al propio pensamiento en cuanto se hacen nuevas exigencias en la lectura de la realidad. La des-parametrización significa privilegiar una postura racional que obliga a fundamentar el discurso epistemológico en la conciencia y en la experiencia de lo constituyente, una clara distinción entre lo que son las lógicas de constitución del discurso y lo que es su comunicación como objetivación textual.

En la perspectiva de la argumentación anterior, es posible reconocer diversidad de planos en los que se puede construir enunciados tales como: primero, el plano del sentido que se corresponde con la colocación ante el mundo, que obliga a un pensamiento no-parametral; segundo, la potencia de la necesidad de realidad, del mundo, que viabiliza la fuerza de lo indeterminado; y tercero, el lenguaje no discursivo como instrumento de una existencia colocada en el mundo que re-actúa ligado con un sujeto que deviene antropológico. En casa uno de estos planos la estructura sujeto-objeto puede revestir modalidades distintas, ya que se avanza desde un predominio de la necesidad de un enunciado a su significado teórico y su sentido práctico. (Zemelman, 1998, p.140)

La cuestión es la necesidad de pensar la mayor implicación de esta capacidad y del uso creativo. Parte de enriquecer la experiencia lingüística, de manera que se puedan incrementar las posibilidades de aprendizaje y el uso creativo del lenguaje (Barthes, 1987). De hecho, el razonamiento constituyente subordina a lo constituido, conformando un espacio de potencialidad de discursos posibles, que pueden ser simultáneos en diferentes niveles de temporalidad y con distintas posibilidades de articulación. Pero es fundamental mantener presente que el inconveniente frente a la percepción no se circunscribe a seguridades, es una cuestión de estar en posibilidad de colocarse ante lo extraño, haciéndolo parte de lo cotidiano. Más importante que emplear la evidencia, es situarse en el campo de estudio del lenguaje para colocarse en el mundo.

El razonamiento consiste en asumir una lógica que corresponda a la potencialidad de la capacidad humana de crecer, de manera que ubica todas sus estructuras desde el lenguaje, en el marco de esta aplicación de la facultad de conocer al hombre. Por esto es que hay que establecer una diferenciación entre la facultad de pensar y la necesidad de pensar. Hay que recuperar los juegos lingüísticos, en cuanto pueda rescatarse en el marco de una lógica de contextualización con una perspectiva de tipo de razonamiento, se trata de estructurar un concepto cuyo significado quede abierto a las alternativas de significaciones posibles. La ampliación de lenguaje es una forma de dar cuenta de las complejidades propias de la construcción de la relación de conocimiento. $Y$ es que

Desde el concepto de logos que rige la relación con la realidad externa al suje- 
to, lo que decimos significa construir el lenguaje pensante, no solamente de comunicación. Pues si el punto de partida es el momento del hombre en su actualidad de presente, significa enfrentarse a sí mismo en los distintos momentos por los que atraviesa en su vida, en virtud de su necesidad de realidad desconocida: he aquí la necesidad de utopía. De lo que resulta que es más importante esta que la verdad, en la medida que impulsa hacer de la época una experiencia posible, y, desde ella, cómo hacer del mundo un contenido de vida. (Zemelman, 1998, p.54)

Por ello es que el sujeto requiere de nuevos lenguajes y de nuevos ángulos de pensamiento, para poder recuperar su relación con el mundo. Romper con los parámetros es la capacidad de resignificación, por lo cual la lógica del ángulo se fundamenta en el movimiento del límite. Lo que entra en conflicto con el lenguaje como parámetro, es que este es claro porque es admitido, ya que escribir es organizar el mundo, es pensarlo. En efecto, el discurso de pensar categorial, a partir del doble movimiento del límite, es el horizonte de posibilidades. Es por eso que el sujeto se enfrenta a dos planos de categorías: la primera está asociada directamente con las condiciones biológicas, como por ejemplo la facultad de lenguaje, y por otro lado es una línea totalmente diferente de razonamiento, el desarrollo históricosocial (Berstein, 1990).
El razonamiento se inicia con una intención cognitiva inicial, es un espacio semántico que funciona de la misma manera que las estructuras conceptuales y su significación asociada con denotaciones. La capacidad de significación es la inclusión de una gnoseología que se puede articular con la intención cognitiva inicial, lo que corresponde con el rompimiento de los parámetros, por lo menos con las determinaciones teóricas. Al tenerse en cuenta los elementos de instalación como colocación, a lo que se hace referencia es a la apropiación del mundo, entonces lo que ocupa en el proceso de constitución de la subjetividad es en gran medida la naturaleza del discurso intelectual. Así que el paso inicial es reconocer el contexto desde el que se define el punto de partida, ya que desde ahí se da lugar a los distintos modos de concreción:

a) de lo externo para transformarlo a partir de la intervención de los sujetos desde los objetos, $y$

b) "la concreción desde la necesidad de sentido que transforma lo real en mundo de vida”. (Zemelman, 2012a p.86)

Los contenidos pasan a ser premisas teóricas como de necesidades de sentido, por ello los contenidos cumplen las condiciones de ser una articulación y articulable a la vez, esto con el objetivo de establecer nuevos parámetros. Entonces no se puede perder la dinámica de lo articulado con lo articulante en el contexto, porque en este se plantean desafíos según 
se den las circunstancias. La discusión se puede desarrollar nuevamente según lo interpretado en el trabajo del profesor Zemelman en dos planos vinculados entre sí: el primero, es el propio de la forma de razonamiento y de su modalidad de argumentación; el segundo, el que concierne a la construcción de enunciados y sus implicaciones en el lenguaje.

Todo esto supone pensar la realidad, no como objeto, ni como problemática, sino como una dimensión trascendente de los límites conceptuales que ya están definidos. Este tipo de razonamientos exige la inclusión de otras realidades en el plano del pensamiento, realidades que son concebibles como requisito epistemológico del mismo razonamiento.

Desde esta perspectiva, la relación con la realidad se desplaza a un ámbito más amplio que trasciende las exigencias puramente cognitivo-formales y posibilita la colocación ${ }^{4}$ que tiene como resultado la constitución del sujeto: la realidad es liberada de las formas socializadas de la racionalidad, de manera que puede reconocer lo que incluye la diversidad, por ello el objeto de la realidad debe permanecer abierto, tanto a la sucesión del movimiento propio como a aquello que lo incluye, y que también puede determinarlo.

4 Es importante entonces aclarar el concepto: a través de la obra del profesor Zemelman se encuentra Colocación como la necesidad de ubicación, posicionamiento incluso de los parámetros que pueden estar por encima de referencias teóricas o normativas.

\section{El análisis del lenguaje en la constitución} del sujeto, del ciudadano: la conciencia histórica y la apertura a lo indeterminado

Preguntar requiere una dinámica vinculante en tanto que rompe con la identidad del tema que se tiene por objeto de trabajo, "ya que la denotación carece de una precisión excluyente" (Zemelman, 2012, p.82), que a su vez impide el distanciamiento de los términos respecto de cualquier significado predeterminado. De lo que se habla, es de la subjetividad que otorga el conocimiento, gracias a que este tiene la capacidad de descubrir las cosas que oculta la realidad. Es importante a la hora de la constitución del sujeto analizar los enunciados, pues la subjetividad se acerca a la realidad a partir del despliegue de la realidad. Pero hay una dificultad que radica en que algún componente no explícito, que esté oculto, se convierte en algo indispensable y su descomposición da sentido a lo que se enuncia.

Ya que los significados no se agotan en los contenidos teóricos, se debe tener claro mientras haya una articulación entre los momentos de posibilidades y los contenidos teóricos que lo conforman que habrá múltiples posibilidades para ir más allá de los límites del código teórico. De aquí que el pensamiento histórico esté por encima de los códigos teóricos, por ello se puede decir que "lo que define la naturaleza problematizadora de la pregunta es poder apropiarse de las consecuencias que se pueden derivar de la teoría acumulada pero desde las necesidades del momento" (Zemelman, 2012, p.83), así pues, las denotaciones nunca están 
cerradas, es decir, en la pregunta entran en tensión lo determinado con lo indeterminado.

Colocarse frente a la realidad que está en permanente movimiento genera el pensar sin límites. Se trata de madurar el movimiento sin el riesgo de sustantivarlo, es decir, con un contenido dado. El predominio de la lógica de determinaciones, refleja el dominio en el desarrollo del conocimiento y de la racionalidad de un tipo de comportamiento del hombre que define su relación con la naturaleza y la realidad sociohistórica. Hay una relación más profunda con la comunicación propositiva que con el proceso de construcción del conocimiento. Esto deja claro que hay que distinguir entre enunciados conformadores de relaciones inclusivas con la realidad, y una forma particular de colocación en, y frente al mundo-realidad.

La colocación propia del sentido del enunciado se corresponde con las proposiciones conformadoras de realidad propositiva con intención transformadora. La apropiación responde a los enunciados de significados que buscan cumplir una función de control, la cual necesita ser comunicada de manera que influya en el pensamiento, para ser socialmente aceptada.

El plano del sentido corresponde a la colocación ante el mundo, a la necesidad de realidad que viabiliza la fuerza de lo indeterminado, del pensar sin sujeción a las exigencias de inteligibilidad, en oposición a un segundo mo- mento donde el pensar y su expresión se amoldan a los requerimientos de la comunicación. Lo fundamental de lo indeterminado consiste en la capacidad de mirar a lo real sin sujeción a límites, conformar a estos límites en forma de estar alertas ante lo indeterminado es una actitud de conciencia que enseña a construir desde lo inédito.

A la luz de lo anterior, la utopía que hace referencia a la posibilidad de un mundo mejor para los sujetos y su constitución, proporciona un marco que ayuda a transformar lo inédito. Para desencadenar la construcción de un lenguaje capaz de crear realidades sin parámetros preestablecidos, hay que hacer uso de la función gnoseológica en torno a la utopía que, conformada por el mundo del hombre caracterizado por la dualidad entre lo que es y el deber ser, pone de manifiesto aquello que se ha querido, y a la vez, tiene en cuenta la existencia de una necesidad de recuperar del pasado aquello que ha dejado insatisfacción.

Estas reflexiones apuntan a transformar la construcción del discurso y de la realidad en lenguajes gestores de existencia y moldeadores de utopías, fundamentales en la constitución del sujeto.

\section{A manera de cierre}

No es extraño escuchar que los procesos de formación y educación institucionalizados no respondan a la realidad y que por lo tanto no se conviertan en ejercicios propios para la constitución de un sujeto consciente de que es 
ciudadano. Por eso es que este artículo pretendió contribuir a la construcción de una entre varias miradas del pensamiento pedagógico latinoamericano, para reivindicar a los sujetos y los movimientos olvidados (Santos, 2009). Y para ello se ha propuesto aquí el lenguaje como elemento fundante del conocimiento, de la apropiación del sentido para instalarse, para colocarse en una conciencia histórica que pone en evidencia un sí mismo del que se apropia de sí como ciudadano. Y todo esto como respuesta a preguntas obligadas como ¿por qué el fracaso de la educación para la formación de la ciudadanía?

En la constitución de la subjetividad y sus procesos de configuración, la formación propiamente dicha parece el resultado de cavilaciones vacías ya que se genera en espacios disciplinarios que invitan a que cada individuo se llene, gracias a que parece que él es un recipiente vacío... y lo llena de contenidos para convertirlo en una especie de ciudadano del primer mundo. Por ello es que este sujeto que se convierte en ciudadano es el resultado de un proceso que tiene sentido si se tiene en cuenta la importancia de clarificar la relación del conocimiento desde la capacidad de incluir niveles de realidad y dinámicas correspondientes a las mismas.

Aquí se han propuesto elementos para la configuración del sujeto que se da forma en la historia que le corresponde de manera contextual y situada en su propia realidad, del que se instala, se coloca allí como ciudadano gracias a sus procesos de subjetivación. Todo esto se encuentra en estrecha relación con la hipótesis planteaba en la tesis doctoral que da origen a este trabajo, y que se remite a la posibilidad de consolidar una vertiente dentro del pensamiento pedagógico crítico latinoamericano, en la medida en que la lectura sistemática y filosófica de autores del pensamiento crítico como Hugo Zemelman lo posibilitan, no solo para una lectura contemplativa, sino como fundamento praxeológico. Por tanto, hay que anotar que el concepto de realidad supera la significación de ser solo la externalidad que parece separada del sujeto, y que el trabajo debe hacerse sobre lo que aparece como objeto de la formación, es decir, la constitución de la subjetividad, de donde emerge el ciudadano de nuestro tiempo.

La realidad deja desde esta perspectiva de ser una cuestión de contenidos, y pasa a ser parte de la articulación entre el contexto y la lectura que el sujeto hace de ella para situarse: esa es la articulación entre lo que vive históricamente y la necesidad de hacerlo suyo para hacerse consciente de sí mismo. Esto es apertura a muchas formas, un arranque que está por encima de lo establecido de manera que supera los límites de lo dado. Estar colocado es hacer suyo un espacio de posibilidades para dejar de ser el objeto determinado por la ciencia. Esto hace que el ciudadano consciente de sí, tenga la capacidad de potenciar y vislumbrar el pensar sobre su propia historia como una dinámica que le permite conocer de manera profunda su situación y la realidad de su contexto, para que no quede atrapado en lo establecido, lo dado. 
Desde el sujeto, y su proceso de configuración, su formación, este trabajo procura reivindicar la ciudadanía que tiene en cuenta a los que han sido invisibilizados, y quiere recoger como reflexión las formas de hacer y de pensar. En último término es una invitación a que la justicia cognitiva sea enfrentada con el uso del respeto por quienes no aparecen como realidades históricas en estos tiempos (Santos, 2009). De hecho, la episteme que sustenta el concepto de sujeto y de ciudadano que se propone, se desarrolló sobre la propuesta de romper con los límites fijados.

Quedó claro en torno al sujeto, que negar lo instaurado es elevarse. Allí, la gnoseología es importante en la construcción del conocimiento y por ende en la configuración del sujeto -la Gnoseología hace análisis de aquellas cuestiones que no han sido llevadas al estatuto científico-. En palabras de Nietzsche "el mundo que nos importa no es un estado de cosas, sino un universo poético y su redondeo una suma infinita de observaciones" (1999, p.161).

\section{Referencias}

Adorno, T.W. (1992). La ideología como lenguaje. Madrid: Taurus Ediciones.

Andrade, L. \& Bedacarratx, V. (2013). "La construcción del objeto de estudio en la obra de Hugo Zemelman: apuntes introductorios". En FOLIOS, Segunda época, (38). Universidad Pedagógica Nacional, Facultad de Humanidades, segundo semestre.
Barthes, R. (1987). El susurro del lenguaje. Más allá de la palabra y la escritura. Barcelona: Paidós.

Benjamin, W. (2008). "Sobre el lenguaje en general y sobre el lenguaje de los hombres". En Ensayos Ecológicos. México: Coyoacán.

Bersntein, B. (1990). La construcción social del discurso pedagógico. Bogotá: Ediciones El Griot.

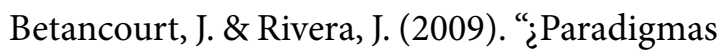
en la pedagogía? O cómo hacer de sí una obra de arte". En Revista El Ágora USB, 9(2). Medellín: Universidad de San Buenaventura.

Betancourt, J. (2012). "Una respuesta al discurso pedagógico de la modernidad: la pedagogía del caos”. En Revista En-Clave Social, 1(1), 42-56. Corporación Universitaria Lasallista.

Betancourt, J. (2013). “Anotaciones para una didáctica no lineal, una que se funda en la potencia del sujeto". En Revista Itinerario Educativo, 27(62). Editorial Universidad de San Buenaventura, Colombia.

De la Garza, E. (2001). "La epistemología crítica y el concepto de configuración”. En Revista Mexicana de Sociología, 63(1), 109-127.

Freire, P. (1971). La educación como práctica de la libertad. Bogotá: Siglo Veintiuno Editores.

Horkheimer, M. (2008). Teoría crítica. Traducción de Edgardo Albizu y Carlos Luis. 
( $3^{\mathrm{a}}$ reimpresión). Buenos Aires: Amorrortu. Biblioteca de Filosofía.

Horkheimer, M. (2010). Crítica de la Razón Instrumental. ( $2^{\mathrm{a}}$ ed.). Madrid: Editorial Trotta.

Koselleck, R. (2012). Historia de conceptos. Estudios sobre semántica y pragmática del lenguaje político y social. Traducción de Luisa Fernanda Torres. Madrid: Editorial Trotta, Verona S.A.

Nietzsche, F. (1999). Éica y teoría de las artes. Madrid: Editorial Tecnós.

Santos, B. de S. (2009). Una Epistemología del Sur. La reinvención del conocimiento y la emancipación social. Buenos aires: Siglo Veintiuno Editores, CLACSO.

Torres, A. (1994). "Experiencias organizativas urbanas y constitución de sujetos sociales". En Movimientos sociales y educación popular Dimensión Educativa. Santafé de Bogotá.
Wulf, C. (2004). Introducción a la antropología de la educación. España: Colección Idea universitaria-educación (Idea Books), primera edición.

Zemelman, H. (1998). Sujeto: existencia y potencia. España: Anthropos Editorial.

Zemelman, H. (2002). Necesidad de conciencia: un modo de construir conocimiento. Traducido por Josetxo Beriain. España: Anthropos Editorial.

Zemelman, H. (2005). Voluntad de Conocer: el sujeto y su pensamiento en el paradigma crítico. España: Anthropos Editorial.

Zemelman, H. (2012a). Pensar y poder: razonar y gramática del pensar histórico. México: Siglo Veintiuno Editores/Universidad de Ciencias y Artes de Chiapas. 